\title{
Comprehensive study of neoadjuvant targeted therapy for resectable non-small cell lung cancer
}

\author{
Yi Bao ${ }^{1 \#}$, Chang Gu${ }^{1 \#}$, Huikang Xie ${ }^{2 \#}$, Shengnan Zhao ${ }^{2}$, Dong Xie ${ }^{1}$, Chang Chen ${ }^{1}$, Gening Jiang ${ }^{1}$, \\ Chenyang Dai ${ }^{1}$, Yuming Zhu $^{1}$
}

${ }^{1}$ Department of Thoracic Surgery, Shanghai Pulmonary Hospital, Tongji University School of Medicine, Shanghai, China; ${ }^{2}$ Department of Pathology, Shanghai Pulmonary Hospital, Tongji University School of Medicine, Shanghai, China

Contributions: (I) Conception and design: Y Zhu, C Dai. (II) Administrative support: Y Zhu. (III) Provision of study materials or patients: Y Bao, C Gu, H Xie. (IV) Collection and assembly of data: Y Bao, C Gu, H Xie. (V) Data analysis and interpretation: Y Bao, C Gu, H Xie. (VI) Manuscript writing: All authors. (VII) Final approval of manuscript: All authors.

"These authors contributed equally to this work.

Correspondence to: Yuming Zhu, MD. Department of Thoracic Surgery, Shanghai Pulmonary Hospital, Tongji University School of Medicine, Shanghai 200443, China. Email: ymzhu2005@aliyun.com; Chenyang Dai, MD. Department of Thoracic Surgery, Shanghai Pulmonary Hospital, Tongji University School of Medicine, Shanghai 200443, China. Email: daichenyang@tongji.edu.cn.

Background: Approximately 30-70\% percent of patients with non-small cell lung cancer (NSCLC) still relapse after receiving complete resection and even suffer distant metastasis. Epidermal growth factor receptor tyrosine kinase inhibitors (EGFR-TKIs) have gradually replaced chemotherapy to become the firstline postoperative NSCLC treatment because they can effectively inhibit the postoperative recurrence of lung cancer. However, the clinical efficacy of neoadjuvant EGFR-TKIs in EGFR-mutant NSCLC patients is still unclear. The purpose of this study was to evaluate their clinical efficacy and to further explore factors affecting recurrence in such patients.

Methods: EGFR-mutated patients receiving neoadjuvant EGFR-TKI treatment in our hospital from July 2016 to September 2020 were retrospectively included. These patients underwent radical tumor resection after treatment. The primary endpoint was the objective response rate (ORR). The secondary endpoints were the major pathological response (MPR), disease-free survival (DFS), and overall survival (OS).

Results: A total of 42 patients who met the inclusion criteria were included in this study. The ORR was $47.6 \%$ (20/42), and the MPR was 23.8\% (10/42). The average follow-up time was 23.7 months. As of the final follow-up date, $18(42.9 \%, 18 / 42)$ patients had experienced tumor recurrence. Of these, there were $11(61.1 \%)$ cases of local recurrence and 7 (38.9\%) cases of distant metastasis, including 5 bone metastases and 2 brain metastases. Multivariate Cox regression results showed that the high-risk subtype $[\mathrm{P}=0.012$, hazard ratio $(\mathrm{HR})=24.560 ; 95 \%$ confidence interval $(\mathrm{CI}), 2.016-299.227$ ] was a risk factor for postoperative recurrence. Patients with a high-risk subtype (solid or micropapillary subtype) had significantly worse recurrence-free survival (RFS, log-rank $\mathrm{P}=0.032$ ). Although patients with pathological remission had a similar RFS as patients who did not achieve pathological remission (log-rank $\mathrm{P}=0.069$ ), the patients without pathological remission showed a tendency toward a worse prognosis.

Conclusions: Neoadjuvant EGFR-TKIs had good effects on EGFR-mutant NSCLC patients at different stages, especially those with MPR. Patients with high-risk subtypes (solid or micropapillary) should be closely followed up after surgery because of the high risk of recurrence.

Keywords: Neoadjuvant targeted therapy; epidermal growth factor receptor (EGFR); objective response rate (ORR); major pathological response (MPR); high-risk subtype

^ ORCID: 0000-0002-8997-2900. 
Submitted Jan 12, 2021. Accepted for publication Mar 25, 2021.

doi: $10.21037 / \mathrm{atm}-21-1134$

View this article at: http://dx.doi.org/10.21037/atm-21-1134

\section{Introduction}

With a high morbidity and mortality, primary lung cancer is the most common malignancy in the world $(1,2)$. Surgical resection is the optimal treatment for early-stage nonsmall cell lung cancer (NSCLC) (3-7). However, 30-70\% of patients still experience recurrence after receiving complete resection and even suffer distant metastasis (8). The 5 -year overall survival (OS) rate of patients with stage II-IIIA NSCLC after lung cancer resection is estimated to be between $65 \%$ and $41 \%$ (9). Death is usually caused by distant metastasis (10), and NSCLC may show undetectable micrometastases (11). Current clinical guidelines recommend cisplatin-assisted regimens for patients with stage II-IIIA NSCLC after complete resection to reduce the likelihood of micrometastasis (12). Postoperative adjuvant therapy can improve the 5 -year OS of patients by $4 \%$ to $8 \%$. Neoadjuvant chemotherapy has also been confirmed in recent years to achieve similar results as adjuvant chemotherapy (13).

The emergence and development of targeted therapy brings hope for the treatment of lung cancer $(8,14,15)$. Epidermal growth factor receptor $(E G F R)$ mutations are common in NSCLC patients in the East Asian population, being present in about half of all patients. Deletions in exon 19 (19DEL) and the L858R mutation are the two most common types of $E G F R$ mutation, accounting for $22.1 \%$ and $20.9 \%$ of EGFR mutations in advanced NSCLC patients, respectively (16). Patients with these mutations respond better to EGFR tyrosine kinase inhibitors (TKIs) than nonmutated patients. The ADJUVANT study compared the efficacy of postoperative administration of gefitinib to postoperative administration of cisplatin plus vinorelbine in resectable NSCLC patients with EGFR mutation. Patients receiving postoperative administration of gefitinib had significantly better disease-free survival (DFS) [28.7 months; 95\% confidence interval (CI), 24.9-32.5] than patients receiving postoperative administration of cisplatin plus vinorelbine (18.0 months; 95\% CI, 13.6-22.3), and the risk ratio was 0.60 (95\% CI, 0.42-0.87; $\mathrm{P}=0.0054)(17)$. Zhang et al. (18) prospectively included 33 patients with resectable stage II-IIIA NSCLC who were sensitive to EGFR-TKIs and who had received gefitinib neoadjuvant therapy to evaluate the safety and feasibility of gefitinib as a preoperative neoadjuvant drug. The patients with a major pathologic response (MPR) after receiving gefitinib neoadjuvant therapy had a better prognosis. Although some studies have investigated the safety and efficacy of EGFRTKIs in neoadjuvant therapy, few studies have analyzed the influence of different pathologies and subtypes on prognosis.

In this study, we retrospectively included 42 patients with EGFR-TKI sensitivity who received preoperative neoadjuvant EGFR-targeted therapy to confirm the clinical efficacy of preoperative neoadjuvant EGFR-TKIs in EGFRmutated NSCLC patients and to explore the effects of different pathological subtypes on prognosis. We present the following article in accordance with the STROBE reporting checklist (available at http://dx.doi.org/10.21037/ atm-21-1134).

\section{Methods}

\section{Inclusion and exclusion criteria}

The inclusion criteria were as follows: (I) age older than 18 years; (II) a deletion in EGFR 19DEL or exon 21 L858R mutation confirmed by pathological diagnosis before operation; (III) preoperative use of neoadjuvant EGFR-TKIs; (IV) Eastern Cooperative Oncology Group (ECOG) score 0 or 1 ; and (V) NSCLC clinical stage after neoadjuvant therapy not higher than stage IIIA (based on the eighth edition of the American Joint Commission on Cancer TNM staging system for NSCLC). The exclusion criteria were as follows: (I) history of cancer; (II) concurrent or sequential use of chemotherapy or other systemic antitumor treatment; (III) EGFR T790 M mutation; and (IV) death from surgical complications.

\section{Treatment plan}

Standard doses of EGFR-TKIs were administered before surgery. After treatment evaluation, surgical resection was performed, including resection of the tumor (surgical procedure depended on the patient's condition) and systemic lymphadenectomy. Postoperative treatment was chosen based on the patient's condition and the clinical experience of the doctor. Usually, platinum-based dual- 
agent chemotherapy was recommended for patients with stage II-IIIA NSCLC as adjuvant therapy.

\section{Response evaluation}

The treatment response was evaluated by computed tomography (CT). CT images of all patients were diagnosed and reviewed by the same group of radiologists. For evaluating the lymph node response after induction therapy, chest computed tomography (CT) and ultrasound-guided fine needle aspiration (EUS-FNA) were all used, taken radiography and cytology together, we could accurately evaluate the status and staging of patients to the utmost extent. At 21 days and 42 days of neoadjuvant therapy, CT imaging of the tumor response to therapy was evaluated based on the Response Evaluation Criteria in Solid Tumors (RECIST, version 1.1). The response was divided into progressive disease (with a diameter increase of no less than $20 \%$ or the occurrence of new lesions), stable disease (SD, diameter change between $-30 \%$ and $+20 \%$ ), partial response (PR, diameter reduction not less than $30 \%)$, and complete remission (CR, imaging results showing no residual disease).

\section{Study outcome}

The primary endpoint of this study was the objective response rate (ORR), which was defined as the percentage of patients who achieved CR or PR according to RECIST version 1.1.10. The secondary endpoints were MPR, DFS, and OS. MPR referred to a major pathological response (MPR), defined as no more than $10 \%$ of the tumor remaining.

\section{Follow-up strategy}

The last follow-up was in November 2019. The followup was completed through outpatient visits or by telephone calls. In the first 3 years after surgery, physical examinations, chest CT scans, ultrasounds of the abdomen/ neck/supraclavicular area, and brain magnetic resonance imaging or CT scans were performed every 6 months. After 3 years, follow-up was performed every 6-12 months. A bone scan was performed every year. OS was defined as the length of time from the day of surgery to death or the last follow-up. RFS was defined as the length of time from the day of surgery to the first recurrence or the last follow-up. To calculate RFS, patients who died of other causes were considered censored subjects without events. Focal recurrence was defined as recurrence at the primary tumor, ipsilateral hilum, ipsilateral mediastinal lymph node, or supraclavicular lymph node, and distant recurrence was defined as recurrence of other sites (19).

The study was conducted in accordance with the Declaration of Helsinki (as revised in 2013). Institutional review board of Shanghai Pulmonary Hospital' approval was granted for this retrospective review along with a waiver of patient consent (No. L20-341-1).

\section{Statistical analysis}

The statistical analysis used SPSS software (version 26.0; IBM Corp., NY, USA). Differences in survival between different subgroups were compared using the log-rank test, and the survival curves were analyzed by the Kaplan-Meier method.

\section{Results}

\section{Baseline information of patients}

We retrospectively included NSCLC patients who underwent preoperative pulmonary biopsy or transbronchial needle aspiration under endobronchial ultrasound between July 2016 and September 2020. Finally, 42 patients who met the inclusion criteria were included in this study. The average age of the patients was 61.6 years (range, 39-76 years old). There were 17 males (40.5\%) and 25 females (59.5\%). The basic clinicopathological information of patients is listed in Table 1. There were 5 (11.9\%), 3 (7.1\%), 22 (52.4\%), $11(26.2 \%)$, and $1(2.4 \%)$ patient(s) at clinical stage IB, IIA, IIIA, IIIB, and IIIC, respectively. Preoperative EGFR detection results showed that 24 (57.1\%), 17 (40.5\%), and 1 (2.4\%) patient(s) had EGFR L858R, 19DEL, and L861Q mutations, respectively. The number of patients using gefitinib ( $n=26)$ was the highest, followed by icotinib $(n=9)$, erlotinib $(n=4)$, and afatinib $(n=4)$. The correspondences between patient subtypes, high-risk subtypes, MPR, and neoadjuvant therapy response are shown in Figure 1.

\section{Primary endpoint}

The ORR in this study was $47.6 \%(20 / 42)$. There was no CR according to imaging, while 5/42 (11.9\%) patients achieved CR according to pathology. All patients underwent complete resection of the tumor after neoadjuvant targeted 
Table 1 Baseline characteristics of patients who received neoadjuvant EGFR-TKIs

\begin{tabular}{|c|c|}
\hline Characteristics & No. of patients (\%) \\
\hline \multicolumn{2}{|l|}{ Sex } \\
\hline Male & $17(40.5)$ \\
\hline Female & $25(59.5)$ \\
\hline Age, years, median (IQR) & $61.6(57.0-67.0)$ \\
\hline Smoking history & $4(9.5)$ \\
\hline FEV1\%, median (IQR) & $86.5(77.1-94.2)$ \\
\hline Blood loss, mL, median (IQR) & $134.8(50.0-100.0)$ \\
\hline Operation time, h, median (IQR) & $2.3(2.0-3.0)$ \\
\hline \multicolumn{2}{|l|}{ cTNM stage } \\
\hline IB & 5 (11.9) \\
\hline IIB & $3(7.1)$ \\
\hline IIIA & $22(52.4)$ \\
\hline IIIB & $11(26.2)$ \\
\hline IIIC & $1(2.4)$ \\
\hline \multicolumn{2}{|l|}{ EGFR mutation } \\
\hline L858R & $24(57.1)$ \\
\hline 19Del & $17(40.5)$ \\
\hline L861Q & $1(2.4)$ \\
\hline \multicolumn{2}{|l|}{ Operative procedure } \\
\hline Lobectomy & $35(83.3)$ \\
\hline Sleeve resection & $2(4.8)$ \\
\hline Bilobectomy & $2(4.8)$ \\
\hline Pneumonectomy & $3(7.1)$ \\
\hline \multicolumn{2}{|l|}{ Side } \\
\hline Left & $18(42.9)$ \\
\hline Right & $24(57.1)$ \\
\hline Resected LN station, median (IQR) & $5.9(5.0-7.0)$ \\
\hline Resected LN count, median (IQR) & $15.3(10.8-21.0)$ \\
\hline VPI & 8 (19.0) \\
\hline STAS & $2(4.8)$ \\
\hline \multicolumn{2}{|l|}{ ypTNM } \\
\hline IA & 14 (33.3) \\
\hline IB & $4(9.5)$ \\
\hline IIB & $4(9.5)$ \\
\hline IIIA & $18(42.9)$ \\
\hline IIIB & $2(4.8)$ \\
\hline
\end{tabular}

Table 1 (continued)
Table 1 (continued)

\begin{tabular}{ll}
\hline Characteristics & No. of patients $(\%)$ \\
\hline Neoadjuvant therapy & \\
Icotinib & $9(21.4)$ \\
Afatinib & $3(7.1)$ \\
Erlotinib & $4(9.5)$ \\
Gefitinib & $26(61.9)$ \\
\hline
\end{tabular}

EGFR-TKIs, epidermal growth factor receptor tyrosine kinase inhibitors.

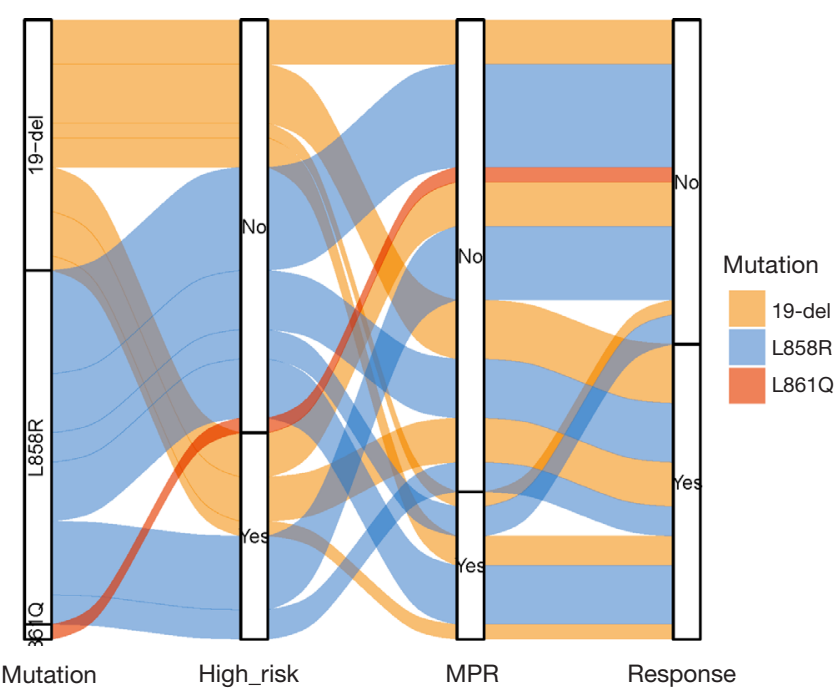

Figure 1 Sankey diagram of the correspondence relationships of mutation type among the 42 patients with neoadjuvant therapy.

therapy. There were 22 patients $(52.4 \%)$ with SD and none with PD. The response rates of all the patients after receiving neoadjuvant targeted therapy are listed in Figure 2.

\section{Secondary endpoint}

In this cohort, the MPR rate was $23.8 \%$ (10/42). The average follow-up time was 23.7 months. The other secondary endpoints of this study were RFS and OS (Figure 3). As of the final follow-up date, 18/42 (42.9\%) patients had tumor recurrence, with an average RFS time of 19.8 months (Figure 4). Among the 18 patients with recurrence, 11 had focal recurrence $(61.1 \%)$ and 7 (38.9\%) had distant metastasis, including 5 patients with bone metastases and 2 patients with brain metastases; 40 patients $(95.2 \%, 40 / 42)$ survived; 2 patients died of lung 


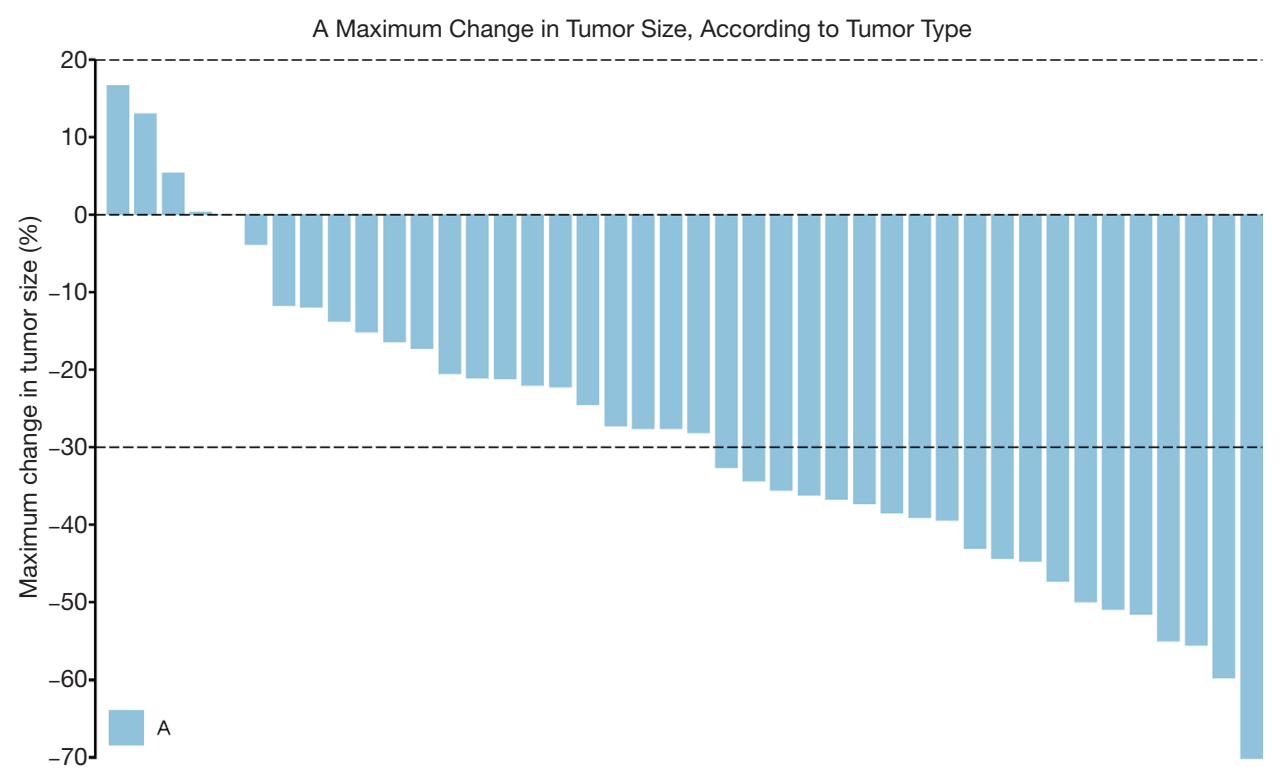

Figure 2 Waterfall plot of the maximum change in tumor size in patients with neoadjuvant therapy. A, adenocarcinoma.

cancer recurrence and metastasis. All patients with stage II disease or above received 4-cycle platinum-based dual-drug chemotherapy or radiotherapy (50-62 Gy/25-28 Fx).

\section{Survival analysis}

Univariate Cox regression analysis showed that the surgical procedure $[\mathrm{P}=0.035$, hazard ratio $(\mathrm{HR})=1.857$; $95 \% \mathrm{CI}$, 1.043-3.306], postneoadjuvant stage (ypTNM) $(\mathrm{P}=0.024$, $\mathrm{HR}=1.393 ; 95 \% \mathrm{CI}, 1.044-1.859)$, pathologic $\mathrm{T}$ category (pT) stage $(\mathrm{P}=0.010, \mathrm{HR}=2.460 ; 95 \% \mathrm{CI}, 1.245-4.858)$, number of nodes dissected $(\mathrm{P}=0.049, \mathrm{HR}=1.098$; 95\% CI, $1.000-1.204)$, and high-risk subtype $(\mathrm{P}=0.038, \mathrm{HR}=2.729$; $95 \%$ CI, 1.055-7.060) were risk factors for postoperative recurrence. We included factors with a $\mathrm{P}$ value less than 0.1 from the univariate Cox regression in the multivariate Cox regression. The results showed that the high-risk subtype $(\mathrm{P}=0.012, \mathrm{HR}=24.560$; 95\% CI, 2.016-299.227) was the only significant risk factor for postoperative recurrence, though ypTNM stage $(\mathrm{P}=0.059, \mathrm{HR}=2.570 ; 95 \% \mathrm{CI}$, $0.964-6.850)$ and pathologic $\mathrm{N}(\mathrm{pN})$ stage $(\mathrm{P}=0.054$, HR $=0.213 ; 95 \% \mathrm{CI}, 0.044-1.024)$ were borderline risk factors for postoperative recurrence (Table 2).

We found that patients with the high-risk subtype (solid or micropapillary subtype) had significantly worse RFS ( $\log$-rank $\mathrm{P}=0.032)$. Although the patients with pathological remission had a similar RFS as the patients who did not achieve pathological remission (log-rank $\mathrm{P}=0.069)$, the patients without pathological remission tended to have a poor prognosis. The EGFR mutant subtype had no effect on the response rate to TKIs in terms of RFS or OS (data not shown).

\section{Discussion}

EGFR-TKIs have been used as the first-line treatment for patients with advanced EGFR mutation NSCLC, but few studies have analyzed the impact of different pathological and mutated subtypes on prognosis. Due to its remarkable efficacy, molecular targeted therapy has been successfully used in the adjuvant treatment of certain types of tumors, such as imatinib for the treatment of gastrointestinal stromal tumors (20,21). Like adjuvant TKIs, neoadjuvant TKIs can function by eliminating micrometastases or residual tumor cells produced by primary tumor cells (possibly with similar genotypes and molecules) (22). Therefore, neoadjuvant TKIs could theoretically reduce disease recurrence and improve OS in NSCLC patients undergoing complete resection.

In this study, we found that preoperative neoadjuvant EGFR-TKIs could be effective in EGFR-TKI-sensitive NSCLC patients. The primary endpoint of ORR was reached by $47.6 \%$ of our cohort. A recent phase II clinical trial of gefitinib for stage II-IIIA NSCLC showed that its ORR reached 54.5\% (18). Xiong et al. (23) have reported that erlotinib, as a neoadjuvant targeted drug for NSCLC 


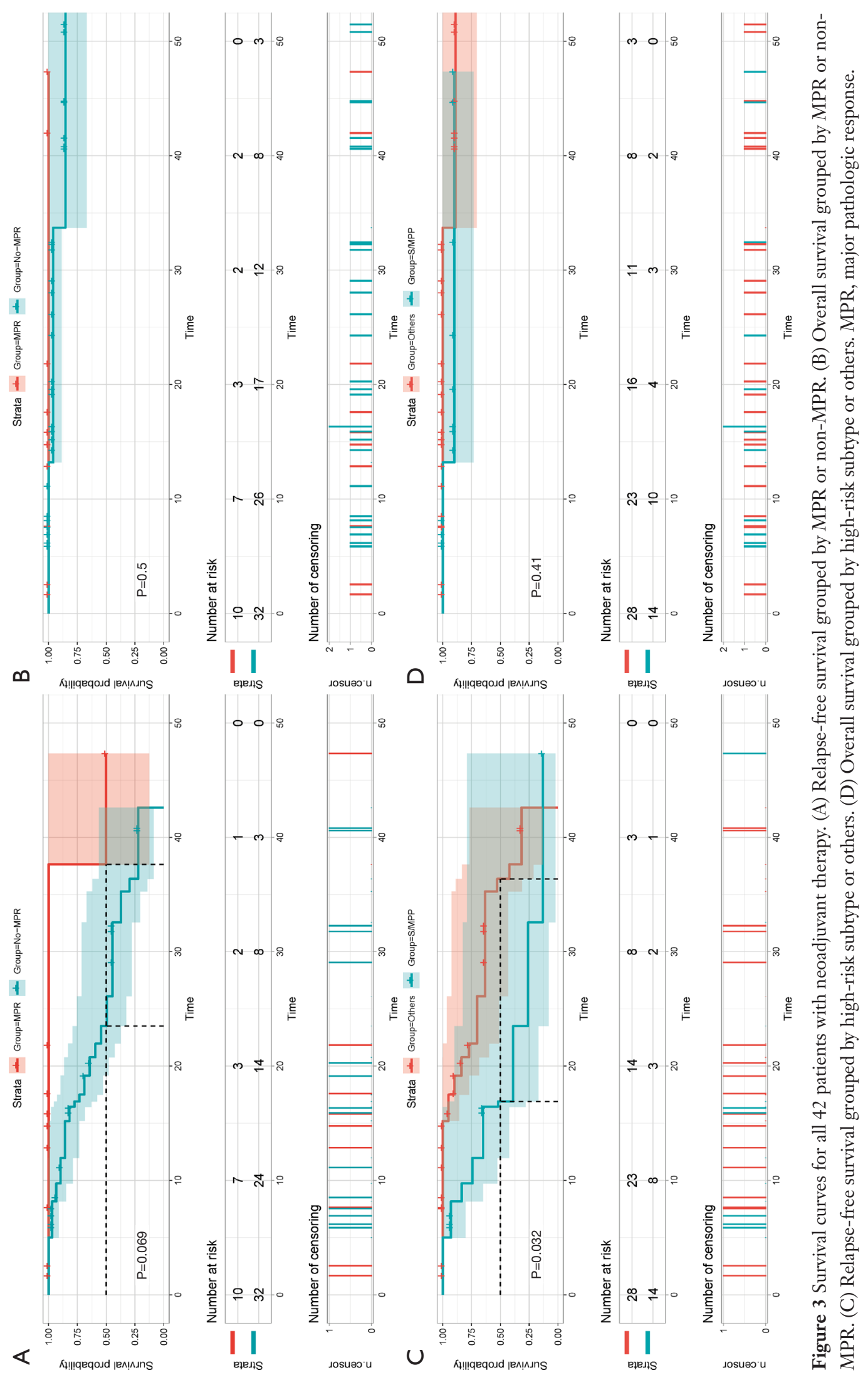




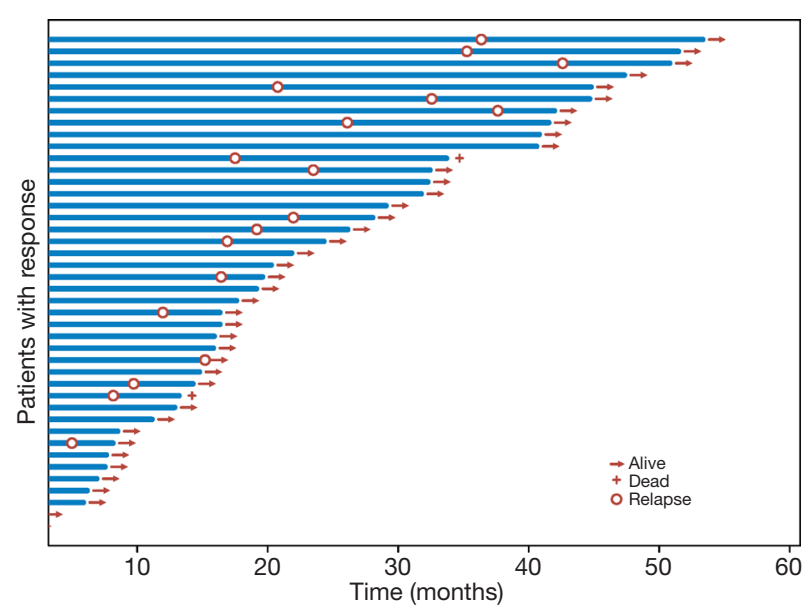

Figure 4 Swimmer plot of all 42 patients with neoadjuvant therapy. patients, achieved an ORR of $42.1 \%$. In the EMERGINGCTONG 1,103 cohort (24), who took erlotinib as neoadjuvant therapy, the ORR was $54.1 \%$. The ORRs of the above studies were consistent with the present one. Possible explanations for our ORR being slightly lower might be as follows: (I) we included patients with clinical stage IB-IIIC, and tumors at different stages have different sensitivities and responsiveness to treatment. Patients with advanced-stage disease are more sensitive to drugs than patients with earlystage disease, so advanced NSCLC patients are more likely to achieve a higher ORR. (II) We included 11 IIIB patients and 1 IIIC NSCLC patient with intratumoral heterogeneity; i.e., tumors containing cell populations with defined molecular and phenotypic characteristics, which make

Table 2 Univariable and multivariable analyses for RFS in patients who received neoadjuvant EGFR-TKIs

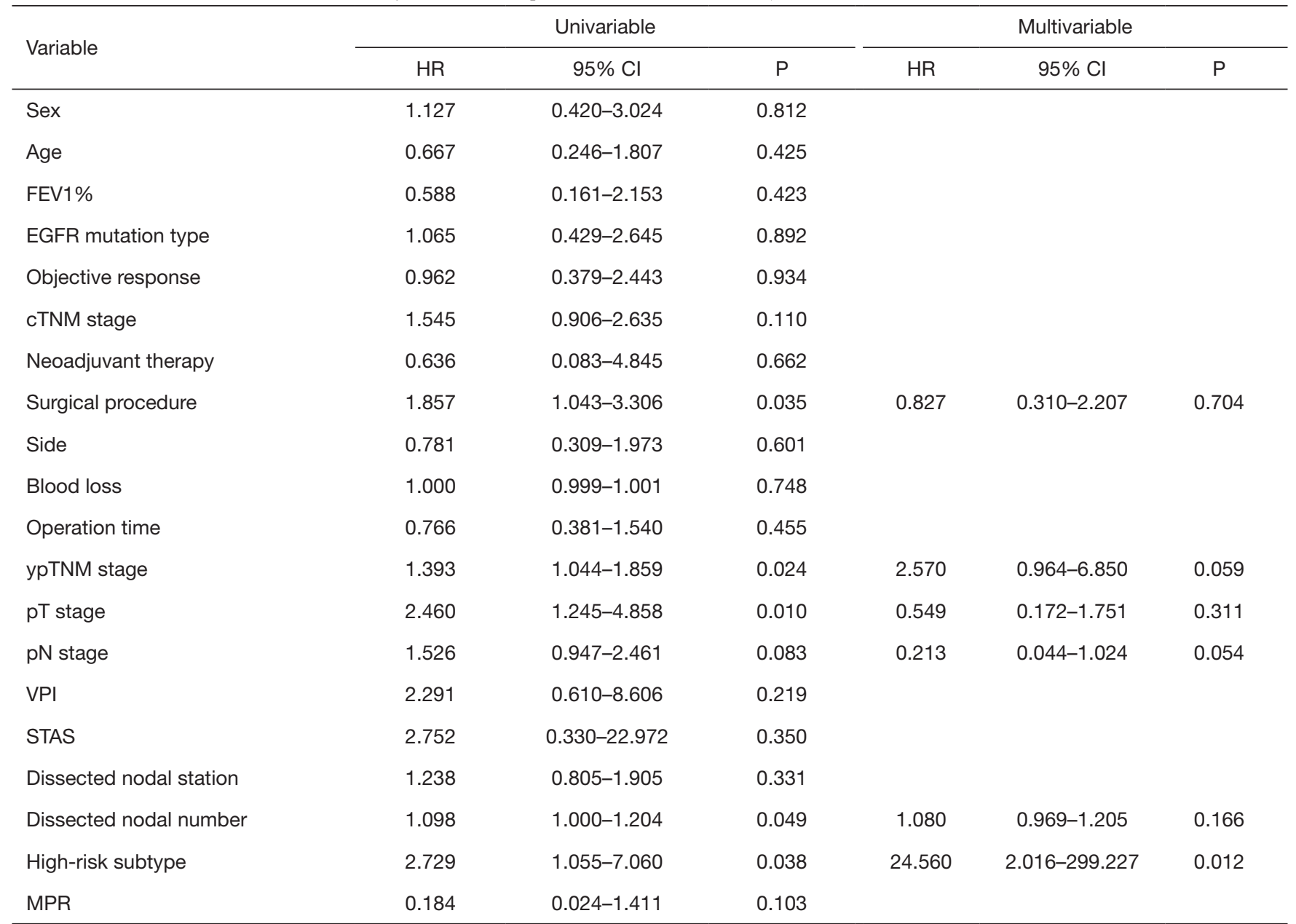

RFS, relapse-free survival; EGFR-TKIs, epidermal growth factor receptor tyrosine kinase inhibitors; HR, hazard ratio; Cl, confidence interval. 
targeted therapy more difficult to implement (22). In lung cancer, with the emergence of driver gene mutations before and after subclonal diversification, evidence of branching evolution has accumulated (25). When wild-type targeted genes emerge, molecular-targeted drugs will not be able to affect these tumor subclones. As the tumor stage progresses, the heterogeneity gradually increases. (III) The above studies mostly examined the effects of specific targeted drugs. This study included a variety of neoadjuvant EGFR-TKIs, which might have generated a bias in ORRs. (IV) Different patients received EGFR-TKIs at different times. The cohort of patients in Xiong et al. (23) took EGFR-TKIs for 56 days preoperatively. Even if the tumor had a certain response to the treatment, the necrotic foci still took some time to absorb the drug after targeted therapy, leading to a less reduced diameter measured on CT.

There are still major controversies regarding preoperative neoadjuvant targeted therapy and adjuvant targeted therapy, especially in terms of patient selection and treatment regimens $(23,24,26,27)$. In most of the neoadjuvant EGFRTKI clinical trials, the included patients were mostly stage IIIA-N2 NSCLC patients with EGFR mutations. In many clinical trials of adjuvant EGFR-TKIs, early-stage lung cancer patients with undetectable EGFR mutations are often included. For example, the EMERGING-CTONG 1,103 (24) clinical trial of neoadjuvant EGFR-TKIs treated patients with gefitinib after surgery, so it is difficult to determine whether the efficacy of preoperative neoadjuvant targeted therapy was better or worse than that of adjuvant targeted therapy. The clinical trial conducted by Zhang et al. (18) included stage II-IIIA NSCLC patients with EGFR mutations, and thus the disease stages included covered a relatively limited range. Our study retrospectively included NSCLC patients with EGFR mutations who were in stage IB-IIIC and who had achieved good efficacy in patients of all stages, confirming the feasibility of neoadjuvant targeted therapy in different stages.

In this study, the MPR of SD patients was $13.6 \%(3 / 22)$, while the MPR of PR patients was 35\% (7/20). These rates explain the effectiveness of imaging in the assessment of targeted therapy for our patients. In many studies, the survival time of patients with PR or CR is better than that of SD or PD patients $(28,29)$. However, the false-negative rate using imaging assessment persists. In this study, the survival time of $\mathrm{PR}$ or CR patients and $\mathrm{SD}$ or PD patients was not statistically significant. The biggest reason may be that even if the tumor has a certain response to the treatment, the necrotic foci still take some time to absorb the targeted therapy drug, leading to a less reduced diameter measured on CT. Besides, drug resistance of EGFR-TKIs is also a major reason. Therefore, the assessment of the effectiveness of neoadjuvant therapy should be combined with preoperative imaging and postoperative pathology.

In the survival analysis, we found that the high-risk subtype $(\mathrm{P}=0.012$, HR $=24.560$; 95\% CI, 2.016-299.227) was a risk factor for postoperative recurrence. The solid or micropapillary subtype of lung adenocarcinoma is a high-risk subtype and a high-risk factor for postoperative recurrence. We found tumor spread through air space (STAS) in this type of patient. Previous studies have also confirmed that STAS is one of the pathological causes of the high recurrence of solid or micropapillary lung adenocarcinoma (30).

This study has the following limitations. (I) The sample was small, and the efficacy of different neoadjuvant EGFRTKIs and the effects of EGFR mutation status on the efficacy of EGFR-TKIs are still unclear. Too few patients were in stage IB, and the efficacy of neoadjuvant EGFRTKIs on NSCLC patients with EGFR sensitivity in stage IB still cannot be determined. (II) The average follow-up time was relatively short. Although some patients had recurrence, the OS of the patients could not be determined. (III) This was a single-center retrospective study. Multicenter external validation is required to confirm our conclusions. (IV) The postoperative treatment regimen of patients relied on the physician's decision based on the patient's condition and the physician's clinical experience, but such decision making may introduce bias to some degree. (V) This is an observational study mainly focused on evaluating the clinical efficacy and further exploring factors affecting recurrence in NSCLC patients, thus the association between biomarkers and tumor prognosis was not discussed in this manuscript, which will be further discussed in the next study.

Neoadjuvant EGFR-TKIs had good effects on NSCLC patients with EGFR mutation in different stages of disease, especially those with MPR. Patients with high-risk subtypes (solid/micropapillary) should be closely followed up after surgery, and the clinician should carefully watch for recurrence.

\section{Acknowledgments}

Funding: This study was supported by funding from the National Natural Science Foundation of China (no. 81802256 and 81902335), the Chen Guang Project supported by the Shanghai Municipal Education 
Commission and Shanghai Education Development Foundation (no. 18CG19), the Outstanding Young Talent Project supported by the Shanghai Pulmonary Hospital (no. FKYQ1907), the Shanghai Rising Star Program (no. 20QA1408300), and the Clinical Research Plan of the SHDC (no. SHDC2020CR4028).

\section{Footnote}

Reporting Checklist: The authors have completed the STROBE reporting checklist. Available at http://dx.doi. org/10.21037/atm-21-1134

Data Sharing Statement: Available at http://dx.doi. org/10.21037/atm-21-1134

Conflicts of Interest: All authors have completed the ICMJE uniform disclosure form (available at http:// dx.doi.org/10.21037/atm-21-1134). All authors report funding from the National Natural Science Foundation of China (no. 81802256 and 81902335), the Chen Guang Project supported by the Shanghai Municipal Education Commission and Shanghai Education Development Foundation (no. 18CG19), the Outstanding Young Talent Project supported by the Shanghai Pulmonary Hospital (no. FKYQ1907), the Shanghai Rising Star Program (no. 20QA1408300), and the Clinical Research Plan of the SHDC (no. SHDC2020CR4028). The authors have no other conflicts of interest to declare.

Ethical Statement: The authors are accountable for all aspects of the work in ensuring that questions related to the accuracy or integrity of any part of the work are appropriately investigated and resolved. The study was conducted in accordance with the Declaration of Helsinki (as revised in 2013). Institutional review board of Shanghai Pulmonary Hospital' approval was granted for this retrospective review along with a waiver of patient consent (No. L20-341-1).

Open Access Statement: This is an Open Access article distributed in accordance with the Creative Commons Attribution-NonCommercial-NoDerivs 4.0 International License (CC BY-NC-ND 4.0), which permits the noncommercial replication and distribution of the article with the strict proviso that no changes or edits are made and the original work is properly cited (including links to both the formal publication through the relevant DOI and the license).
See: https://creativecommons.org/licenses/by-nc-nd/4.0/.

\section{References}

1. Miller KD, Fidler-Benaoudia M, Keegan TH, et al. Cancer statistics for adolescents and young adults, 2020. CA Cancer J Clin 2020;70:443-59.

2. Gu C, Huang $Z$, Chen $X$, et al. TEAD4 promotes tumor development in patients with lung adenocarcinoma via ERK signaling pathway. Biochim Biophys Acta Mol Basis Dis 2020;1866:165921.

3. Gu C, Wang R, Pan X, et al. Sublobar resection versus lobectomy in patients aged $\leq 35$ years with stage IA nonsmall cell lung cancer: a SEER database analysis. J Cancer Res Clin Oncol 2017;143:2375-82.

4. Gu C, Wang R, Pan X, et al. Comprehensive study of prognostic risk factors of patients underwent pneumonectomy. J Cancer 2017;8:2097-103.

5. Chen J, Gu C, Chen X, et al. Clinicopathological and prognostic analyses of 86 resected pulmonary lymphoepithelioma-like carcinomas. J Surg Oncol 2021;123:544-52.

6. Gu C, Huang Z, Dai C, et al. Prognostic Analysis of Limited Resection Versus Lobectomy in Stage IA Small Cell Lung Cancer Patients Based on the Surveillance, Epidemiology, and End Results Registry Database. Front Genet 2018;9:568.

7. Gu C, Pan X, Chen Y, et al. Short-term and mid-term survival in bronchial sleeve resection by robotic system versus thoracotomy for centrally located lung cancer. Eur J Cardiothorac Surg 2018;53:648-55.

8. Yuan Y, Huang Q, Gu C, et al. Disease-free survival improved by use of adjuvant EGFR tyrosine kinase inhibitors in resectable non-small cell lung cancer: an updated meta-analysis. J Thorac Dis 2017;9:5314-21.

9. Goldstraw P, Chansky K, Crowley J, et al. The IASLC Lung Cancer Staging Project: Proposals for Revision of the TNM Stage Groupings in the Forthcoming (Eighth) Edition of the TNM Classification for Lung Cancer. J Thorac Oncol 2016;11:39-51.

10. Lou F, Sima CS, Rusch VW, et al. Differences in patterns of recurrence in early-stage versus locally advanced nonsmall cell lung cancer. Ann Thorac Surg 2014;98:1755-60; discussion 1760-1.

11. Zhu E, Xie H, Gu C, et al. Recognition of filigree pattern expands the concept of micropapillary subtype in patients with surgically resected lung adenocarcinoma. Mod Pathol 2020. [Epub ahead of print]. doi: 10.1038/s41379-02000711-8. 
12. Postmus PE, Kerr KM, Oudkerk M, et al. Early and locally advanced non-small-cell lung cancer (NSCLC): ESMO Clinical Practice Guidelines for diagnosis, treatment and follow-up. Ann Oncol 2017;28:iv1-iv21.

13. NSCLC Meta-analysis Collaborative Group. Preoperative chemotherapy for non-small-cell lung cancer: a systematic review and meta-analysis of individual participant data. Lancet 2014;383:1561-71.

14. Gu C, Shi X, Dai C, et al. RNA m6A modification in cancers: molecular mechanisms and potential clinical applications. Innovation (N Y) 2020;1:100066.

15. Jiao D, Yang S. Overcoming Resistance to Drugs Targeting KRASG12C Mutation. Innovation (N Y) 2020;1:100035.

16. Shi Y, Au JS, Thongprasert S, et al. A prospective, molecular epidemiology study of EGFR mutations in Asian patients with advanced non-small-cell lung cancer of adenocarcinoma histology (PIONEER). J Thorac Oncol 2014;9:154-62.

17. Zhong WZ, Wang Q, Mao WM, et al. Gefitinib versus vinorelbine plus cisplatin as adjuvant treatment for stage II-IIIA (N1-N2) EGFR-mutant NSCLC (ADJUVANT/ CTONG1104): a randomised, open-label, phase 3 study. Lancet Oncol 2018;19:139-48.

18. Zhang Y, Fu F, Hu H, et al. Gefitinib as neoadjuvant therapy for resectable stage II-IIIA non-small cell lung cancer: A phase II study. J Thorac Cardiovasc Surg 2021;161:434-2.e2.

19. Gu C, Pan X, Wang R, et al. Analysis of mutational and clinicopathologic characteristics of lung adenocarcinoma with clear cell component. Oncotarget 2016;7:24596-603.

20. Dematteo RP, Ballman KV, Antonescu CR, et al. Adjuvant imatinib mesylate after resection of localised, primary gastrointestinal stromal tumour: a randomised, doubleblind, placebo-controlled trial. Lancet 2009;373:1097-104.

21. Corless CL, Ballman KV, Antonescu CR, et al. Pathologic and molecular features correlate with long-term outcome after adjuvant therapy of resected primary GI stromal tumor: the ACOSOG Z9001 trial. J Clin Oncol 2014;32:1563-70.

Cite this article as: Bao Y, Gu C, Xie H, Zhao S, Xie D, Chen C, Jiang G, Dai C, Zhu Y. Comprehensive study of neoadjuvant targeted therapy for resectable non-small cell lung cancer. Ann Transl Med 2021;9(6):493. doi: 10.21037/atm-21-1134
22. Zhang J, Fujimoto J, Zhang J, et al. Intratumor heterogeneity in localized lung adenocarcinomas delineated by multiregion sequencing. Science 2014;346:256-9.

23. Xiong L, Li R, Sun J, et al. Erlotinib as Neoadjuvant Therapy in Stage IIIA (N2) EGFR Mutation-Positive Non-Small Cell Lung Cancer: A Prospective, Single-Arm, Phase II Study. Oncologist 2019;24:157-e64.

24. Zhong WZ, Chen KN, Chen C, et al. Erlotinib Versus Gemcitabine Plus Cisplatin as Neoadjuvant Treatment of Stage IIIA-N2 EGFR-Mutant Non-Small-Cell Lung Cancer (EMERGING-CTONG 1103): A Randomized Phase II Study. J Clin Oncol 2019;37:2235-45.

25. de Bruin EC, McGranahan N, Mitter R, et al. Spatial and temporal diversity in genomic instability processes defines lung cancer evolution. Science 2014;346:251-6.

26. Goss GD, O'Callaghan C, Lorimer I, et al. Gefitinib versus placebo in completely resected non-small-cell lung cancer: results of the NCIC CTG BR19 study. J Clin Oncol 2013;31:3320-6.

27. Kelly K, Altorki NK, Eberhardt WE, et al. Adjuvant Erlotinib Versus Placebo in Patients With Stage IBIIIA Non-Small-Cell Lung Cancer (RADIANT): A Randomized, Double-Blind, Phase III Trial. J Clin Oncol 2015;33:4007-14.

28. Mandrekar SJ, An MW, Meyers J, et al. Evaluation of alternate categorical tumor metrics and cut points for response categorization using the RECIST 1.1 data warehouse. J Clin Oncol 2014;32:841-50.

29. Takeda M, Okamoto I, Nakagawa K. Survival outcome assessed according to tumor response and shrinkage pattern in patients with EGFR mutation-positive nonsmall-cell lung cancer treated with gefitinib or erlotinib. J Thorac Oncol 2014;9:200-4.

30. Dai C, Xie H, Su H, et al. Tumor Spread through Air Spaces Affects the Recurrence and Overall Survival in Patients with Lung Adenocarcinoma $>2$ to $3 \mathrm{~cm}$. J Thorac Oncol 2017;12:1052-60.

(English Language Editor: J. Gray) 